\title{
FUNCIONES DE LOS TRABAJADORES SOCIALES DEL ÁREA DE LA SALUD EN LOS HOSPITALESY CLÍNICAS DE TERCERO Y CUARTO NIVEL DE LA CIUDAD DE MEDELLÍN, COLOMBIA*
}

\section{FUNCTIONS OF SOCIAL WORKERS IN THE HEALTH FIELD IN HOSPITALS AND CLINICS OF THIRD AND FOURTH LEVEL OF THE CITY OF MEDELLÍN, COLOMBIA}

\author{
Marta Elena Correa- Arango** \\ Alejandra Corena-Argota ${ }^{* * *}$ \\ Camila Chavarriaga-Estrada**** \\ Karla García-Valencia***** \\ SARA USME-BAENA******
}

\section{Resumen}

Objetivo. Este artículo pretende dar a conocer las funciones desarrolladas por la profesión de Trabajo Social en el ámbito de la salud, específicamente en clínicas y hospitales de la ciudad de Medellín. Metodología. Basado en un estudio cualitativo-descriptivo que recolectó la información mediante entrevista semiestructurada, diseñada por expertos y aplicada a 11 trabajadores sociales en ocho clínicas y hospitales. El análisis posterior de la información se realizó utilizando el software ATLAS.ti. Resultado. El estudio encontró que los trabajadores sociales tienen una perspectiva integral de la salud, lo cual permite caracterizar su participación allí como integral y sistémica: su intervención está centrada en los pacientes y sus redes de apoyo familiar y social. Conclusión. Los trabajadores sociales cumplen un significativo papel integrador en equipos interdisciplinarios, lo cual contribuye a la accesibilidad e integralidad del servicio de salud, a solucionar problemas conexos a la salud física y la humanización de la asistencia.

Palabras clave: trabajo social, funciones profesionales, clínicas, hospitales.

\footnotetext{
* Este artículo es resultado de una investigación denominada "Funciones de Trabajador Social en Salud", estudio cualitativo descriptivo, realizado por el grupo de investigación GITS de la Facultad de Trabajo Social de la Universidad Pontificia Bolivariana.

** Universidad Pontificia Bolivariana.E-mail: martaelena.correa@gmail.com

(D) orcid.org/0000-0002-4402-1888 Google Scholar

*** Universidad Pontificia Bolivariana. E-mail: mayra.corena@upb.edu.co

(D) orcid.org/0000-0003-0020-9181 Google Scholar

**** Universidad Pontificia Bolivariana. E-mail: maria.chavarriaga@upb.edu.co

(1) orcid.org/0000-0002-9784-6293 Google Scholar

***** Universidad Pontificia Bolivariana. E-mail: karla.garcia@upb.edu.co

(D) orcid.org/0000-0002-0641-0065 Google Scholar

***** Universidad Pontificia Bolivariana. E-mail: sara.usmeba@upb.edu.co

(1) orcid.org/0000-0001-5360-7322 Google Scholar
} 
Funciones de los trabajadores sociales del área de la salud en los hospitalesy clínicas de tercero y cuarto nivel de la ciudad de Medellín, Colombia

\begin{abstract}
Objective. This paper aims to publish the functions developed by the Social Work profession in the field of health, specifically in clinics and hospitals of the city of Medellin. Methodology. Based on a qualitative-descriptive study, in which information was collected through a semi-structured interview designed by experts and applied to eleven social workers in eight clinics and hospitals. The subsequent analysis of the information was done using ATLAS.ti software. Results. The study found that social workers have a comprehensive perspective on health, which allows characterizing their participation there as integral and systemic: their intervention is centered on patients and their family and social support networks. Conclusion. Social workers fulfill a significant integrating role in interdisciplinary teams, which contributes to the accessibility and integrality of the health service to solve problems related to physical health and the humanization of care.
\end{abstract}

Key words: social work, professional functions, clinics, hospitals.

\title{
Introducción
}

El trabajo social es una profesión que se caracteriza por su complejidad y su diversidad, se ocupa tanto del individuo como de la sociedad, de los grupos, de las familias y de las comunidades, no solo en cuanto a aspectos sociales sino también económicos y de desarrollo. Se trata de una disciplina que con el paso del tiempo ha adquirido importancia y reconocimiento en las dinámicas presentes del mundo social, gracias al continuo trabajo y esfuerzo de parte de sus profesionales.

Tal como lo plantea la Escuela Nacional de Trabajo Social de la Universidad Nacional Autónoma de México (2017), el trabajo social:

(...) es una disciplina que, mediante su metodología de intervención, contribuye al conocimiento y a la transformación de los procesos sociales, para incidir en la participación de los sujetos y en el desarrollo de las potencialidades de las personas a partir de la interacción social de estas. (párr.1)

El trabajo de esta profesión se reconoce en los diferentes ámbitos que conforman la sociedad, pero su intervención en Colombia, en lo que comprende al área de la salud, requiere mayor estudio y sistematización. No sobra referirse a la importancia del trabajo social en el sector de la salud, aunque en general, se considera que esta profesión brinda un aporte significativo desde su perspectiva del conocimiento y manejo de los procesos de salud-enfermedad de las personas. Se valora positivamente su propósito de contribuir al bienestar de los individuos siendo un garante de los derechos y deberes del paciente, máxime cuando la concepción 
de salud y enfermedad es entendida como el resultado de factores biológicos, psicológicos, materiales, sociales, ambientales, culturales, y de funcionamiento y organización de los seres humanos.

Sin embargo, al revisar las publicaciones que al respecto hacen referencia a Colombia, se halla un vacío significativo de información en cuanto a la comprensión de la profesión en el ejercicio de sus funciones dentro del sistema de salud y, específicamente, en las instituciones de más alta complejidad dentro del mismo, denominados clínicas y hospitales con servicios de tercero y cuarto nivel.

Para la profesión resulta relevante el ejercicio de conocimiento y sistematización de su desempeño profesional en el ámbito de la salud, ya que le permite fortalecer su estructura teórico-metodológica como propuesta de intervención social. Si bien el trabajo social ha sido de tradición práctica-técnica, se propone que, sin perder esa fortaleza, mediante la investigación científica, se potencie para fortalecer la construcción de un saber reflexivo. En este sentido, la Facultad de Trabajo Social de la Universidad Pontificia Bolivariana se constituye como promotora de la investigación y, desde su concepción humanista de la educación, asume mediante el trabajo de grado que da origen a este artículo, un papel protagónico y responsable en el nivel de calidad académica que logre la profesión, aportando conocimientos encaminados a desarrollar propuestas metodológicas y modelos de formación que posibiliten a los profesionales su contribución para que la vida social humana se dignifique.

Para efectos de este trabajo, se entiende la función como el conjunto de actividades que reflejan todos aquellos problemas que un profesional debe o debería ser capaz de enfrentar o resolver, es decir, "el tipo de actividad laboral que caracteriza e identifica el trabajo socialmente útil que realiza el hombre para alcanzar un objetivo determinado" (Portuondo como se citó en Torres, 2008, párr. 6).

Con la finalidad de analizar las necesidades presentes de la sociedad y entrever las futuras, el trabajador social realiza unos procesos y tareas de intervención para el adecuado ejercicio de sus funciones, dentro de la cuales se encuentran: la intervención directa que se realiza en diversos ámbitos de desempeño y precisa de un contacto personal entre el profesional y la persona, familia o grupo de implicados - de tal forma que la relación que se establece entre el trabajador social y el sistema cliente (individuos, familia, grupo pequeño) es un elemento significativo en el cambio de las situaciones problemáticas-; actividades de estudio, análisis, sistematización, planificación, evaluación, coordinación y supervisión; además de la intervención directa, el trabajador social desarrolla también intervenciones indirectas de mayor cobertura que soportan el ejercicio de las directas, como son la formulación de programas y proyectos, la investigación social, la formulación de políticas sociales y su ejecución, más la administración de programas y servicios sociales. 
Según Vásquez (como se citó en Ballestero, Viscarret y Úriz, 2013), se han señalado las siguientes funciones en las diferentes áreas profesionales:

Atención directa. Mediante la detección y tratamiento psicosocial de las necesidades sociales individuales, grupales, familiares y de la comunidad, los trabajadores sociales gestionan los recursos humanos y organizativos, contribuyendo así a la administración de las prestaciones de responsabilidad pública, su distribución y la protección de las poblaciones.

Planificación y evaluación (la primera no es posible sin la segunda y viceversa) en los macro y microsistemas el trabajador social a partir del conocimiento y análisis de procesos sociales y necesidades, participa en el diseño, el desarrollo y la ejecución de planes, programas y proyectos sociales. Así como en nivel estratégico-prospectivo mediante la formulación de las políticas sociales.

Gerencia y administración. El trabajador social trabaja en la gerencia y administración de los servicios sociales, a través de la organización, dirección y coordinación de los mismos.

Investigación y docencia. El trabajador social desarrolla la investigación inherente a su propio trabajo profesional para revisarlo permanentemente y buscar nuevas formas de enfocar y afrontar los problemas vitales a los que debe dar respuesta. También debe realizar procesos sistematizados para el estudio de la realidad con un método y unos procedimientos de cientificidad para así aportar a la contextualización de las problemáticas. Por otra parte, el trabajador social aumenta su conocimiento de la realidad social mediante la investigación para poder modificar las prácticas sociales que crean desigualdad e injusticia social y, por lo tanto, condicionan el desarrollo autónomo de los sujetos.

Por medio de este artículo se dan a conocer algunos aspectos relativos a las funciones de los trabajadores sociales, encontrados en la investigación realizada en ocho clínicas y hospitales del sistema de salud ${ }^{1}$ en la ciudad de Medellín (Colombia), caracterizando las funciones que realizan los trabajadores sociales en las instituciones de salud de alta complejidad, es decir, aquellas donde se realizan intervenciones de salud de tercero y cuarto nivel (Ministerio de Salud de la República de Colombia, 1994).

\footnotetext{
${ }^{1}$ El sistema de salud en Colombia tiene como eje central el Sistema General de Seguridad Social en Salud (SGSSS), el cual supone una afiliación al sistema que es obligatoria y que se hace a través de las entidades promotoras de salud (EPS), públicas o privadas, las cuales reciben las cotizaciones y, a través de las instituciones prestadoras de servicios (IPS), ofrecen el Plan Obligatorio de Salud (POS). Hay dos regímenes de aseguramiento para dar cobertura a toda la población: el régimen contributivo (RC) y el régimen subsidiado (RS), vinculados entre sí a través de un fondo de recursos llamado Fondo de Solidaridad y Garantía (FOSYGA). (Guerrero, Gallego, Becerril y Vásquez, 2011, p. 145).
} 


\section{Metodología}

Este artículo es resultado de un estudio cualitativo de carácter descriptivo basado en métodos de recolección de datos sin medición numérica, cuyo alcance final es comprender las funciones del trabajador social en el área de salud, específicamente en clínicas y hospitales de Medellín donde se prestan servicios de tercero y cuarto nivel. Fue realizado a partir de una recolección de datos durante dos semestres, con 11 profesionales de trabajo social que laboran en ocho clínicas u hospitales de alta complejidad.

Para el desarrollo de la investigación se diseñó una entrevista a profundidad con la ayuda de expertos para recolectar la información requerida, que al aplicarse individualmente a cada persona fuente de información, fue grabada, posteriormente transcrita, codificada y luego analizada con el apoyo de ATLAS.ti.

La muestra se fue seleccionando mediante la referenciación de profesionales de trabajo social de las clínicas y hospitales, hasta la saturación de categorías. Se accedió a la localización de las fuentes de información a través de una lista de hospitales y clínicas que fue facilitada vía telefónica por la Secretaría Seccional de Salud de Antioquia, requiriendo a las instituciones (clínicas y hospitales de alta complejidad en Medellín) los datos relativos a la profesional o profesionales que laboraban allí; se solicitó a cada uno de los posibles entrevistados la lectura y firma de un consentimiento informado, en el cual se garantizaba la participación voluntaria y la confidencialidad de la información; posteriormente se realizó la codificación, clasificación y análisis de información apoyados por el software ATLAS.ti.

\section{Resultados}

\section{Funciones de trabajadores sociales en salud}

En cuanto al desarrollo de las funciones del trabajador social en instituciones de salud de alta complejidad, a través de la entrevista semiestructurada que se llevó a cabo con 11 profesionales, se encontraron los siguientes elementos:

\section{Concepción de salud y enfermedad}

Resulta pertinente establecer la concepción que los trabajadores sociales tienen respecto al binomio salud-enfermedad que, de una u otra forma, delimita y sirve de marco al desempeño de estos profesionales en este ámbito. Al respecto, se encontró que tienen una perspectiva integral multicausal en la cual la salud y la enfermedad están definidas por muchos factores que interactúan entre sí: desde lo biológico, hasta elementos del entorno social, económico, cultural, el estilo y los hábitos de vida de las personas y los grupos sociales. 
Funciones de los trabajadores sociales del área de la salud en los hospitalesy clínicas de tercero y cuarto nivel de la ciudad de Medellín, Colombia

Ejemplo de esta postura integral son respuestas como esta que se obtuvo de una de las TTSS entrevistadas y que representa una concepción prácticamente unánime entre las TTSS participantes en el estudio:

\begin{abstract}
(...) la salud está definida por factores individuales (edad, sexo, herencia, escolaridad, estilos de vida), familiares (dinámica familiar, relaciones interpersonales, comunicación), sociales (acceso a los servicios de salud, orden público, acceso a los medios de transporte), económicos (ingresos, tenencia de la vivienda, condiciones laborales) y culturales (costumbres, creencias). (Entrevista 7, comunicación personal, 16 de septiembre de 2016)
\end{abstract}

Una concepción integral del binomio salud-enfermedad significa una ampliación de perspectiva, donde a lo biomédico se suma la dimensión psicosocial, lo cultural y lo espiritual como expresiones de la existencia humana, y es desde aquí donde se hace pertinente el aporte de las ciencias sociales y específicamente del Trabajo Social al conocimiento y la intervención en el campo de la salud y la enfermedad.

También se indagó por qué, a juicio de los trabajadores sociales entrevistados, era necesario un profesional del trabajo social en salud, específicamente en las clínicas y hospitales. Al respecto, resultó claro lo siguiente: el trabajador social en el ámbito de la salud tiene un rol fundamental a la hora de lograr una mirada global, estructural y sistémica de la situación de salud y enfermedad de las personas, su familia y las comunidades; esto representa un aporte a la reflexión permanente en torno a las condiciones estructurales que generan enfermedad, así como la consideración de todos aquellos factores protectores que contribuyen a garantizar la salud y una mejor calidad de vida de la población. El fortalecimiento de la participación social en salud y la valoración de las perspectivas preventivas y promocionales para la conservación de la salud que son norte para la acción política, planificadora y educativa del trabajo social dan pleno sustento a la necesidad de su presencia allí; el acompañamiento del profesional en trabajo social no es solo vital para la comunidad de usuarios, sino también para los equipos de salud que atienden problemáticas específicas en esta área.

Las TTSS reconocieron la importancia del papel que realiza el trabajador social en el área de la salud, en el marco de una concepción integral de la misma, y señalaron perspectivas metodológicas y estrategias a partir de las cuales realizan su aporte, tal como se plantea en estas afirmaciones de una de las TTSS entrevistadas:

En el área de salud, el Trabajo Social desarrolla procesos de actuación en estos ámbitos denominados métodos: intervención individual y familiar, intervención grupal y, finalmente, intervención comunitaria. De esta manera el trabajador social es capaz de identificar las necesidades y 
problemáticas sociales que afectan la salud de las personas, hogares y comunidades, desarrollando su labor no solo al interior de las instituciones de salud, sino en espacios donde tienen lugar las relaciones sociales y se generan redes de apoyo que soportan y ayudan a la persona enferma. La salud, desde un concepto integral del ser humano, está determinada por diferentes factores, entre ellos el contexto social, familiar, económico, ambiental y cultural que rodea a las personas. El trabajador social puede identificar los factores de riesgo y los factores protectores que rodean al individuo, familia y comunidades, interviniendo de diversas formas en la prevención y control de las múltiples enfermedades físicas y mentales. (Entrevista 7, comunicación personal, 16 de septiembre de 2016)

Las familias representan mucho más que cuidado y apoyo mutuo; para muchos seres humanos constituyen el espacio en el que se desarrollan las más profundas experiencias existenciales: intimidad, identidad e individualidad, memoria del pasado y la esperanza en el futuro, porque los más profundos sentimientos humanos tienen su fuente en la familia. En las entrevistas se constató que la intervención con las familias es percibida por los profesionales como particularmente importante en el desarrollo de su desempeño en salud, pues la familia es el espacio por excelencia de socialización y apoyo frente al cuidado de la misma y la atención a la enfermedad. La siguiente respuesta presenta una postura común entre las TTSS entrevistadas:

\begin{abstract}
(...) el trabajo social es importante en la medida en que desde la formación que se tiene en ese campo, se posee una habilidad para orientar, acompañar y asesorar a las familias. En el ámbito hospitalario es necesario que haya una persona que tenga conocimiento en la parte familiar, que tenga la habilidad de identificar las capacidades que tienen los sujetos para potenciarlas, que pueda propiciar espacios de escucha y comunicación tanto de las necesidades del paciente como de la familia y la información médica que tienen para proporcionar, es decir, es necesario porque se presenta como un puente en un equipo interdisciplinario donde se vela por los derechos que tiene el paciente. (Entrevista 4, comunicación personal, 25 de agosto de 2016)
\end{abstract}

El trabajador social en el área de la salud se caracteriza por el apoyo y acompañamiento a los pacientes, a su familia y entorno, al igual que a los miembros del equipo interdisciplinario, trabajando sobre recursos internos y externos que facilitan el proceso de recuperación de la salud, identificando en primer lugar las necesidades sociales y problemáticas familiares y sociales que han afectado a ésta. Pero no solo integrando el aspecto familiar, también buscando tener impacto en las comunidades, desarrollando estrategias donde se permita socializar y generar redes de apoyo que serán de utilidad para la recuperación del paciente en lo físico, social, familiar, etc., tal como lo señala una de las entrevistadas: "Mi papel en la institución de salud 
Funciones de los trabajadores sociales del área de la salud en los hospitalesy clínicas de tercero y cuarto nivel de la ciudad de Medellín, Colombia

en la que trabajo es movilizar recursos internos y externos del paciente (familia, comunidad de residencia, redes institucionales) que aporten en el mejoramiento de la condición de enfermedad del paciente"(Entrevista 7, comunicación personal, 16 de septiembre de 2016).

La familia genera procesos que afectan la rehabilitación o el curso de la propia enfermedad, dependiendo de la actitud que esta asuma frente al diagnóstico y el cuidado de la misma. La participación del trabajador social en el apoyo al grupo familiar, y en el espacio interinstitucional para que este responda adecuadamente a los derechos y necesidades del paciente, se evidencia en la respuesta planteada por una de las profesionales entrevistadas:

\begin{abstract}
Nosotros trabajamos aquí con familia; yo pienso que la enfermedad es como (...) una crisis inesperada que se da al interior de una familia. Cuando está acá la familia con su paciente enfermo, todas esas problemáticas que había en su núcleo familiar, que son común o normales, aquí se agudizan mucho más y esa es la importancia de nosotros estar acá para apoyar a las personas en el afrontamiento de estas situaciones, los tratamientos a veces son muy prolongados, entonces la familia se agota y a eso también hay que sumarle el contexto en el que estamos, las dificultades con las entidades aseguradoras que también hace que esos tratamientos no sean de la mejor manera, porque no hay adherencia, porque no les dan los medicamentos. (Entrevista 5, comunicación personal, 1 de septiembre de 2016)
\end{abstract}

El objetivo que se persigue con esta modalidad de trabajo es involucrar a las familias del usuario que está atravesando un problema de salud, proporcionándole información sobre la enfermedad y sobre el tratamiento, propiciando el medio para desarrollar habilidades de manejo de la enfermedad y brindando el apoyo que dichas familias necesiten para hacer frente a este problema e incluso, a asumir de la manera más sana posible la realidad de la muerte. Otra de las TTSS entrevistadas dice:

\begin{abstract}
Brindo apoyo de tipo familiar (...) de tipo terapéutico en procesos de elaboración de pérdidas, también trabajamos los duelos tanto duelo anticipado, como duelo congelado, el duelo inmediato, todos esos duelos los trabajamos porque aquí tenemos una población muy mayor. (Entrevista 2, comunicación personal, 12 de agosto de 2016)
\end{abstract}

\title{
Diagnóstico social
}

Por otra parte, la información que han aportado las TTSS entrevistadas acerca de los elementos y el proceso realizado en la elaboración del diagnóstico social, se observa que este cumple un papel fundamental como eje que orienta las intervenciones que hace el profesional en el 
desarrollo del trabajo directo con personas, grupos, familias y comunidades en el área de salud.

A lo largo de la historia del trabajo social, el diagnóstico social ha sido un concepto asumido como elemento fundamental de la intervención social: es un eje transversal del proceso o tratamiento de la enfermedad que padece la persona; va más allá del análisis o evaluación socio familiar y demográfico; se elabora y se implementa con el propósito de conocer mejor una situación y su contexto para actuar y así lograr tomar decisiones concretas e implementar estrategias adecuadas. Los diagnósticos, al igual que las necesidades, no son estáticos ya que cambian o se modifican en la medida en que el sujeto interactúa, se moviliza, y el medio social recibe y devuelve la presión ejercida por la persona al modificar también sus condiciones primarias. Los diagnósticos son evolutivos y sitúan el acento en la interrelación y mutua influencia persona-sociedad.

Desde una perspectiva sistémica, el trabajador social identifica características o condiciones sociales que están relacionadas con el surgimiento o persistencia de alteraciones, crisis en la salud integral individual o del grupo familiar o social, y facilita estos elementos al equipo interdisciplinario para la discusión, análisis y definición de las acciones a emprender.

Las entrevistas realizadas en hospitales y clínicas también permitieron identificar que la elaboración y presentación del diagnóstico forma parte vital del papel del trabajador social: en la fase inicial de la atención se implementa para facilitar la comprensión de la situaciónproblema, para fundamentar una atención integral por parte de los equipos interprofesionales, para determinar las acciones requeridas y priorizar las situaciones y personas de mayor riesgo o con limitaciones de mayor alcance, lo que constituye, sin duda, un ejercicio reflexivo que fundamenta el actuar profesional y va construyendo un cuerpo teórico, metodológico y profesional, posibilitando verdaderas transformaciones sociales y humanas en la población atendida.

\section{Equipos interprofesionales}

Con relación a las funciones que cumplen las TTSS entrevistadas dentro de los equipos interprofesionales, esto fue lo que se encontró:

Los equipos interprofesionales en salud responden a una concepción integral del proceso salud-enfermedad; son grupos conformados por profesionales de diversas áreas en los cuales se genera un intercambio de saberes orientado por el propósito común de servir más y mejor al sujeto en condición de enfermedad para que recupere su salud y la preserve.

En los equipos interprofesionales en salud, el trabajador social se orienta principalmente a tratar aquellas problemáticas sociales, económicas, familiares y culturales que influyen en el proceso de salud-enfermedad; a brindar al sujeto y su familia el apoyo, la información y el 
Funciones de los trabajadores sociales del área de la salud en los hospitalesy clínicas de tercero y cuarto nivel de la ciudad de Medellín, Colombia

asesoramiento requerido sobre los recursos sociales y de salud existentes y su uso; a desarrollar procesos educativos que les permitan a las personas tomar conciencia sobre su responsabilidad personal en su salud; a acompañarlos en la toma de decisiones frente a sus situaciones de salud y a favorecer el desarrollo de su propia autonomía. Respecto al trabajo de los equipos interprofesionales, dos entrevistadas manifiestan lo siguiente:

\section{(...) en el quipo interprofesional el TS tiene a su cargo la evaluación socio familiar, todo lo que tiene que ver con el contexto familiar y social es manejado por trabajo social... somos el puente de comunicación del hospital con otras entidades sociales como bienestar familiar (...) y también considero que somos un puente entre la familia y el grupo interdisciplinario. (Entrevista 5, comunicación personal, 1 de septiembre de 2017)}

"El diagnóstico social es muy valorado en la institución. El concepto del trabajador social se comparte con el equipo tratante y se tiene en cuenta durante la hospitalización y en el plan de egreso del paciente" (Entrevista 7, comunicación personal, 16 de septiembre de 2016). En general, se puede afirmar que en los grupos interdisciplinarios los trabajadores sociales aportan una concepción del ser humano desde su complejidad, viéndolo como un ser bio-psicosocial.

\section{Participación ciudadana}

La participación ciudadana se ha convertido últimamente en un asunto central y de actualidad en el debate político, civil, profesional y académico en general, y con un significado sustantivo entre los profesionales vinculados con las políticas de bienestar social, entre ellos el trabajo social. Se asume que una parte muy importante de los problemas de salud están asociados a determinantes sociales, por lo tanto, solo las personas, las comunidades y los grupos sociales podrán con un accionar consciente y reflexivo, modificar estas situaciones que generan obstáculos o deterioran sus condiciones de salud.

En el trabajo social existe la conciencia de que son múltiples los factores que favorecen una participación efectiva en salud. Se evidencia el papel de la información como una variable clave en la participación, por lo que es crucial generar estrategias informativas y educativas. Se rescata la importancia de la participación de abajo hacia arriba y promovida desde lo local. La voz de las entrevistadas lo confirma:

Aquí trabajamos en dos líneas: línea de proyección, en la cual realizamos programas dirigidos a la participación comunitaria; en estos se encuentran los programas de formación a cuidadores 
familiares. Es un programa que se creó acá en la clínica para capacitar a familiares de pacientes en algunos temas de cuidado, para cuando el paciente egrese el familiar esté formado. También es un programa de responsabilidad social porque el paciente se le muere o se alivia y esa persona está capacitada y la podemos llamar de acá de la clínica para que cuide pacientes y puede tener una retribución. Y la línea de educación, que consiste en una estrategia que genera procesos educativos al interior de la institución; entonces aquí damos charlas en las salas de espera, se reparten algunos folletos de charlas que se organizan, se habla de los deberes y derechos a los pacientes hospitalizados y al grupo familiar. (Entrevista 5, comunicación personal, 1 de septiembre de 2016)

\section{Investigación en trabajo social}

Como en todas las profesiones, cuando un trabajador social en el ejercicio profesional cotidiano está en presencia de un hecho que no conoce, se plantea algunas interrogantes. La actuación mediante la cual conoce estas situaciones, siguiendo un proceso sistemático que supone unos referentes teóricos y el desarrollo de una actividad metódica se llama investigación, la cual no es ajena a los procesos de la profesión y, además, le define un marco ético.

La investigación, en relación a la aplicación de resultados, va dirigida tanto al diseño de modelos de intervención como a unos logros prácticos; así, la investigación en trabajo social es un procedimiento reflexivo, sistemático, controlado y crítico que tiene por objetivo analizar aspectos de la realidad social con una expresa finalidad práctica: la transformación de una situación problemática. La investigación debe aportar elementos fundamentales y suficientes que expliquen una realidad determinada para programar la intervención.

El interés por conocer cómo las trabajadoras sociales en salud han participado en actividades investigativas se vio entorpecido por la visión completamente formal según la cual las TTSS perciben una separación entre la intervención y la investigación, por lo tanto, señalaron que su participación en este ámbito es escasa y que las clínicas y hospitales poco apoyan la participación de trabajo social en estos procesos estrictamente formales.

Con relación a la participación de las trabajadoras sociales entrevistadas en la realización de investigaciones en salud, solo una de las entrevistadas refirió esta experiencia: "Ya estamos culminando una investigación sobre los cuidadores de los pacientes que nos permita comprender las necesidades de los cuidadores y cómo prevenir los posibles deterioros de salud de esta población” (Entrevista 3, 14 de agosto de 2016). 
Funciones de los trabajadores sociales del área de la salud en los hospitalesy clínicas de tercero y cuarto nivel de la ciudad de Medellín, Colombia

\section{Políticas en salud}

Respecto a la participación de los TTSS en la formulación y ejecución de políticas en salud, las entrevistas realizadas permiten evidenciar la participación de dicha disciplina en el área de la ejecución de políticas de salud, mediante las tareas diarias realizadas por los profesionales. Sin embargo, en general los TTSS no trabajan directamente con la construcción o formulación de políticas en salud, ya que dentro de los espacios de toma de decisiones no se cuenta con la participación de esta profesión. El trabajo social surge como un canal de recolección y transmisión de información hacia esos grupos de trabajo encargados de la planeación de dichas políticas, y es así como contribuye a la construcción de las mismas.

Las trabajadoras sociales entrevistadas se encuentran laborando en instituciones cuyo énfasis está en la atención restaurativa o curativa, por lo tanto, lo que realizan en materia de prevención se dirige a evitar algunos problemas que se generan como secuelas de la enfermedad, como también a situaciones que se agravan por la falta de adherencia al tratamiento y a sus indicaciones. Ellas perciben que su papel de prevención y promoción de la salud está orientado hacia la educación, de manera que permite evitar dificultades sucesivas o mayores que se pueden desencadenar a partir de la situación de salud que en el momento se atiende. Así se expresan al respecto:

Hacemos promoción y prevención con el grupo de atención al menor en riesgo.Yo considero que casi siempre se hace educación, promoción y prevención que aunque llegan acá por una situación "se me quemó con el agua de una olla" son accidentes prevenibles, son accidentes que pueden pasar en un segundo, no son situaciones netamente de descuido, entonces es necesario empezar a educar, aunque se educa en cosas que ya pasaron, pero se educa para que no vuelvan a pasar; educar respecto a conseguir espacios seguros, en las barandas, en las ventanas (...) Es dar esa información y de hecho cuando se identifican madres adolescentes, madres primerizas que no tienen mucho soporte de su familia extensa también se les da mucha educación, desconocen muchas cosas, entonces se les enseña para prevenir accidentes. (Entrevista 8, comunicación personal, 20 de septiembre de 2016)

Respecto a las responsabilidades que tienen los trabajadores sociales en la administración de los servicios de salud, encontramos la siguiente información: la administración es un proceso mediante el cual se busca facilitar que los sujetos que conforman una sociedad reciban servicios de calidad (en este caso en el ámbito de la salud), es decir, servicios más accesibles y ágiles, que cuenten con recursos para dar la orientación necesaria respecto a los problemas conexos a la 
situación de salud física que presente el paciente y en los que se aporte información suficiente y pertinente a la población atendida; servicios donde se instauren relaciones adecuadas entre usuarios y el personal administrativo y de salud; relaciones empáticas caracterizadas por la amabilidad, calidez, respeto y confidencialidad, donde se instaure una comunicación respetuosa y directa que facilite la atención de las quejas y opiniones de los usuarios.

Los trabajadores sociales realizan aportes significativos en el ámbito administrativo, pues desarrollan gestiones para la coordinación interinstitucional que facilitan la accesibilidad e integralidad del servicio de salud. Una de las trabajadoras sociales señaló al respecto: "Activo rutas de atención interinstitucional para la remisión de los casos que lo ameriten” (Entrevista 2, comunicación personal, 12 de agosto de 2016).

En lo relativo a contribuir a la solución de problemas conexos a la situación de salud física que presente el paciente y a la humanización de la asistencia, una de las TTSS señaló:

\begin{abstract}
La asistencia es fundamental (...) no solamente se debe enfocar en la parte de salud del paciente; para mí es muy importante en el área de oncología porque los pacientes con esta enfermedad necesitan esa red de apoyo para saber qué medicamentos consumía, sus antecedentes de salud. Si el paciente llega acá solo y no está el trabajador social para ayudarle a ubicar esa familia, ese paciente se queda solo durante la estancia hospitalaria. Es de suma importancia reforzarle a los acompañantes la compañía, porque se ha identificado que los pacientes tienen mayor recuperación cuando están con sus familiares que cuando están solos; ese amor, ese cariño, ese buen trato mientras están ahí, eso es lo que les ayuda a salir adelante. (Entrevista 3, comunicación personal, 14 de agosto de 2016)
\end{abstract}

Con relación al papel de los trabajadores sociales respecto a contribuir al mejoramiento de la accesibilidad a los servicios, esto planteó una de las entrevistadas:

Cuando llegan las jornadas de salud que el hospital realiza allá en los pueblos, la gente nos dice: "Llevo años esperando que me autoricen un examen por mi EPS y no he podido”. Nosotros, ¿qué hacemos? Lo revisamos, le damos una orden y con el hospital del municipio transcriban esta orden para que la EPS lo atienda. (Entrevista 3, comunicación personal, 14 de agosto de 2016)

Lo anterior demuestra la importancia del papel del TS en la cualificación de los servicios de salud y, por consiguiente, su aporte a la administración de los mismos desde esta perspectiva. 
Funciones de los trabajadores sociales del área de la salud en los hospitalesy clínicas de tercero y cuarto nivel de la ciudad de Medellín, Colombia

\section{Planeación frente a las intervenciones en salud}

Según la literatura revisada, "La planificación forma parte de la práctica profesional de las trabajadoras sociales" (Barranco y Herrera, 2009, p. 37). Esta se puede desarrollar en tres niveles: micro-social, que comprende el diseño de tratamientos, intervenciones y proyectos sociales (planeación operativa); meso (planeación programática); y macrosocial (planeación estratégica). Estas últimas comprenden el diseño de programas, planes de desarrollo y servicios sociales (Medina, 2009).

En cuanto a la planeación frente a las intervenciones y actividades correspondientes a su cargo, la mayoría de las profesionales realizan una planeación operativa: cuentan con un cronograma y plan de trabajo que en algunas ocasiones es elaborado y desarrollado en coordinación con diversos profesionales, realizando así un trabajo interdisciplinario con el fin de detectar, controlar y decidir sobre diferentes situaciones o casos puntuales que se presenten en la institución. Esta planeación que se realiza en el contexto de un trabajo interdisciplinario se hace con el fin de lograr una visión más integral, desde las diferentes profesiones y disciplinas, de la atención en salud, tiene una perspectiva local o regional y a corto plazo. En la voz de una de las entrevistadas:

Yo planeo los programas que yo manejo; entonces yo diría que yo estoy en
todas las etapas del ciclo PHVA (planificar, hacer, verificar y actuar),es
decir, yo debo planificar mis acciones, yo las hago y las ejecuto, no solo
yo, pero en la planificación siempre soy yo, en el hacer yo ya estoy
mucho más acompañada con médicos; mi rol es muy interdisciplinario,
entonces yo me muevo con otras áreas del hospital, en verificar también
estoy yo con otras áreas; cuando hablo de control y monitoreo de mis
programas o proyectos y en el actuar o verificar también estoy con otras
áreas, pero en todas las estructuras estoy yo liderando, obviamente a
nivel interdisciplinario. (Entrevista 3, comunicación personal, 14 de
agosto de 2016)

También los trabajadores sociales participan en la formulación de proyectos que presentan a agencias internacionales para efectos de cofinanciación:

En el montaje de proyectos participo elaborando propuestas mediante la metodología de marco lógico para la búsqueda de financiadores y para eso se deben elaborar los proyectos o se deben actualizar a una matriz internacional alemana, que es la matriz de marco lógico para solicitar dinero de cooperación internacional; es decirles a otras agencias de cooperación, venga, ayúdenos que estamos aportando a mejorar los niveles de salud y la calidad de vida de una población. (Entrevista 5, comunicación personal, 1 de septiembre de 2016). 
Este testimonio permite identificar que las profesionales en trabajo social que participaron de esta investigación efectivamente realizan la función de planificación.

\section{Conclusión}

A medida que se obtiene una mayor claridad acerca de que las causas de la enfermedad tienen relación con el sujeto y los entornos sociales, se comprende mejor la necesidad de abordar estos asuntos de manera integral para lograr un conocimiento y acción que resulte eficiente y eficaz.

Por lo tanto, es necesario considerar la determinación social de la enfermedad. Se ha documentado que la organización económica y social de la sociedad en que se vive y el desarrollo y estructura de las formas productivas en las cuales la población labora definen unas formas predominantes de enfermar, como señala Echeverri (2011): "Se ha demostrado la vinculación que existe entre trabajo y salud: el tipo de inserción que tienen los individuos en el mercado de trabajo se relaciona directamente con formas específicas de enfermar y morir" (p. 52).

Esta perspectiva de concepción integral y multicausal de la salud se encuentra vigente en los trabajadores sociales consultados para este estudio y, en general, en la literatura revisada que desarrolla la relación trabajo social-salud (Gil, 2007; Consejo Nacional de Trabajo Social, 2014). Se reconoce la importancia de la participación del profesional del trabajo social en salud, labor que se consolida a través del aporte que este profesional hace de una mirada global, integral y sistémica de la situación de salud y enfermedad de las personas, su familia y las comunidades; planteamiento que se encuentra presente también en diversos trabajos científicos que se revisaron (Castillo y Abad, 1992; Rodríguez, Loor y Anchundia, 2017; Montecinos, 2011).

En los procesos de atención directa en salud el trabajador social cumple un papel preponderante: aporta al equipo el tratamiento de los factores sociales tanto en lo que se refiere al diagnóstico de los mismos como a la intervención en ellos; aporta el conocimiento y manejo de los recursos sociales de las instituciones de salud y los recursos vinculados a las redes de servicios externas, tales como servicios sociales, centros y servicios de salud especializados, educación, etc. (Ituarte, 1992, 2012).

Al revisar otros trabajos de investigación realizados respecto a la temática del desempeño de los trabajadores sociales dentro de equipos interprofesionales en salud, encontramos que estos responden a una concepción integral del proceso salud-enfermedad y en ellos, el trabajador social tiene a su cargo la atención de problemáticas sociales "mediante el estudio y tratamiento de los factores socio familiares que condicionan la salud individual y familiar” (Laucirica, 2004, 
Funciones de los trabajadores sociales del área de la salud en los hospitalesy clínicas de tercero y cuarto nivel de la ciudad de Medellín, Colombia

p. 4). En estos equipos la gestión de trabajador social facilita el acercamiento al paciente y su familia al informar y asesorar específicamente sobre los recursos sociales y en salud existentes y su correcto uso, también brinda un acompañamiento en el acceso a los mismos, más el trabajo de concientización de los pacientes y de sus redes de apoyo familiar sobre su autoresponsabilidad en su salud, y el soporte en la toma de decisiones para la solución de sus problemas que posibilite el desarrollo de su autonomía personal (Rodríguez, 2007).

Los trabajos referenciados presentan perspectivas similares respecto a los trabajadores sociales, a las señaladas por las personas entrevistadas, con lo cual se concluye que los trabajadores sociales en los grupos interdisciplinarios favorecen una concepción del ser humano desde su complejidad, viéndolo como un ser multidimensional e integral.

En su ejercicio profesional cotidiano y en la observación de la experiencia de las personas frente a los servicios de salud, los trabajadores sociales perciben un doloroso desencuentro entre la atención en salud y los requerimientos psico-sociales y humanos de las personas, por lo que han asumido la participación ciudadana como un asunto central y de actualidad en el debate en torno a la salud. El papel de la participación ciudadana no radica solo en el hecho de la contribución que esta puede hacer a unos mejores y más adecuados servicios de salud, sino también al fortalecimiento de las capacidades de las personas y de las comunidades para hacerse cargo y protagonizar la solución de sus asuntos. Además, es necesario considerar el criterio de asumir la participación de manera sustantiva, promoviendo el acceso real de la ciudadanía a la elaboración, ejecución y evaluación de políticas y programas, mediante los procesos de participación que se promueven desde el trabajo social (Pastor, 2009).

Respecto al papel desarrollado por trabajo social en el área de investigación, tal como explicita Parola (2009), es menester la articulación de investigación e intervención como dos instancias necesarias para una formación profesional que toma como eje la cuestión social de hoy. La autora sostiene que:

El sentido de la producción de conocimiento en la profesión es permitir
a los profesionales construir conocimientos desde la cotidianidad,
desarrollar destrezas, habilidades, opciones valóricas que tienen más
bien una relación con un tipo de racionalidad práctica que con una
racionalidad teórica. (Parola, 2009, p. 38)

Si bien las trabajadoras sociales entrevistadas realizan ejercicios sistemáticos, con sustento teórico y de utilidad práctica de acercamiento y conocimiento de la realidad, no perciben estas tareas como investigación; por lo tanto, señalaron que es muy escasa su participación en el ámbito formalmente establecido para este efecto (grupos de investigación, proyectos de investigación) y que las clínicas y hospitales apoyan muy poco la participación de trabajo social en estos procesos. 
Esto resulta especialmente paradójico si se tiene en cuenta lo que plantea Miguel Miranda al referirse a los orígenes del trabajo social, señalando que el Departamento de Sociología de Chicago, a la sazón, el primer departamento de sociología americano creado a principios del siglo XX, en el que nació el American Journal of Sociology, la American Sociological Society, "se servía para sus monografías del rico material de primera mano que habían recogido las trabajadoras sociales" (Miranda, 2003, p. 15).

Si bien los trabajadores sociales entrevistados participan en su quehacer diario en la ejecución de políticas en salud y sus experiencias constituyen un mecanismo de recolección de información que eventualmente es enviada hacia los grupos de trabajo que participan de la formulación y planeación de dichas políticas, no se presenta con frecuencia la participación directa del trabajador social en la construcción de las mismas, ni su vinculación amplia con instancias y procesos políticos en los cuales se toman decisiones.

De acuerdo con la información recolectada de las profesionales de trabajo social en el ámbito de la salud, se encontró que la mayoría de las profesionales realizan una planeación operativa, que se realiza en el contexto de un trabajo interdisciplinario y tiene una perspectiva local o regional y a corto plazo.

Finalmente, se plantea que las funciones del trabajador social podrían verse enriquecidas y fortalecidas a medida que el profesional tenga una mayor formación afín con el área de salud, de manera que pueda trascender el modelo asistencial hacia una participación más amplia en el diseño de programas de promoción y prevención en salud, en el diseño y formulación de políticas públicas, y en la realización de investigaciones en salud.

\section{Referencias}

Ballestero, A., Viscarret, J.J. y Úriz, M.J. (2013). Funciones profesionales de los trabajadores sociales en España. Cuadernos de Trabajo Social, 26(1), 127-138. Recuperado de http:// revistas.ucm.es/index.php/CUTS/article/view/41664/39715

Barranco, C. y Herrera, J. (2009). Planificación estratégica y trabajo social. Trabajo social y salud, (62), 37-62.

Castillo, A. y Abad, G. (1992). Las funciones del trabajador social en los Equipos de Atención Primaria del Insalud. Cuadernos de Trabajo Social, (4-5), 129-138. Recuperado de: https: / / revistas.ucm.es/index.php/CUTS/article/viewFile/CUTS9192110129A/8558

Consejo Nacional de Trabajo Social. (2014). Trabajo Social en el Sistema de Salud Colombiano. Perfiles y competencias básicas del trabajador social. Recuperado de https://www.minsalud. 
Funciones de los trabajadores sociales del área de la salud en los hospitalesy clínicas de tercero y cuarto nivel de la ciudad de Medellín, Colombia

gov.co/sites/rid/Lists/BibliotecaDigital/RIDE/VS/TH/Trabajo\%20Social\%20en\%20 el\%20Sistema\%20de\%20Salud\%20Colombiano_Octubre2014.pdf

Echeverri, J. (2010). Relaciones entre las ciencias sociales y el campo de la salud. Revista de Psicología Universidad de Antioquia, 2(4), 47-55. Recuperado de http://aprendeenlinea. udea.edu.co/revistas/index.php/psicologia/article/view/10429/12058.

Escuela Nacional de Trabajo Social de la Universidad Autónoma de México. (Sin Fecha). ¿Qué es el Trabajo Social? Recuperado de http: / /www.trabajosocial.unam.mx/queestsocial.html

Gil, D. (2007). En el margen o marginado: el lugar epistemológico del trabajo social en salud. Trabajo social hoy, 2, 57-66.

Guerrero, R., Gallego, A., Becerril, V. y Vásquez, J. (2011). Sistema de salud de Colombia. Salud Publica México, 53 (2), 144-155. Recuperado de http://www.scielosp.org/pdf/ $\mathrm{spm} / \mathrm{v} 53 \mathrm{~s} 2 / 10 . \mathrm{pdf}$

Ituarte, A. (1992). Procedimiento y proceso en trabajo social clínico. Madrid, España: Siglo XXI.

Ituarte, A. (2012). Cuestiones básicas en el proceso clínico de Atención Psicosocial. Trabajo social y salud, (72), 5-16.

Laucirica, C. (2004). Profesionalidad y relaciones interprofesionales en las ciencias de la salud. Revista Bioética, 5 (1), 4-7. Recuperado de http: / /www.cbioetica.org/revista/41/410407.pdf

Medina, J. (2009). Las funciones básicas de la planificación. Recuperado de https:/ /www.cepal. org/ilpes/noticias/paginas/6/.../c2_teoria_de_las_decisiones.ppt

Ministerio de Salud de la República de Colombia. (1994). Resolución número 5261 de 1994. Recuperado de http: / / www.defensoria.gov.co/public/pdf/11/salud/r5261_94.pdf

Miranda, M. (2003). Pragmatismo, Interaccionismo Simbólico y Trabajo Social. De cómo la caridad y la filantropía se hicieron científicas (tesis de doctorado). Universitat Rovira i Virgili, Tarragona, España.

Montecinos, X. (2011). Rol del trabajador Social en la Salud en Chile. Entrevista a Ximena Montecinos. [Entrada de blog]. Recuperado de http://trabajadoressociales.bligoo.cl/ content/view/1129357/ROL-DEL-TRABAJADOR-SOCIAL-EN-LA-SALUD-ENCHILE-Entrevista-a-Ximena-Montecinos.html\#.WeQHZmiCzIW

Parola, R.N. (2009). Producción de conocimiento en el trabajo social: una discusión acerca de un saber crítico sobre la realidad social. Buenos Aires, Argentina: Editorial Espacio.

Pastor, E. (2009). El trabajo social en la dimensión de las políticas públicas de proximidad: responsabilidad y compromiso social. Humanismo y trabajo social, 8, 85-109. Recuperado de http://buleria.unileon.es/xmlui/bitstream/handle/10612/1500/Hum8_art3. pdf?sequence $=1$ 
Rodríguez, L., Loor, L. y Anchundia, V. (2017). Las funciones del trabajador social en el campo de salud. Revista Contribuciones a las Ciencias Sociales. Recuperado de http: / /www.eumed. net/rev/cccss/2017/02/portoviejo.html

Rodríguez, M.A. (2007). Organización y Funcionamiento del Trabajo Social Sanitario en Atención Primaria de Salud. Recuperado de http://www.trabajosocialbadajoz.es/colegio/wpcontent/uploads/2011/05/TRABAJO-SOCIAL-SANITARIO_OK.pdf

Torres, M. (2008). Metodología para definir funciones profesionales. Revista Cubana de Salud Pública, 34 (4). Recuperado de http://scielo.sld.cu/scielo.php?script $={ }_{\text {sci }}$ arttext\&pid $=$ S0864-34662008000400017\&lng $=$ es\&tlng $=$ es. 